\title{
Exclusión económica y social de la educación en \\ América Latina: una historia sin fin
}

\author{
Ibarra Rosales Esther \\ Universidad Montrer \\ mibarra33@hotmail.com
}

\section{Resumen}

Diversos organismos internacionales (CEPAL, 2010, Banco Mundial, 2010 y Banco Interamericano de Desarrollo, 2016) ubican a América Latina como la región más desigual del mundo, cuyos efectos se traducen de manera importante en la educación. Cierto que, en términos generales, los países latinoamericanos registran avances significativos en la enseñanza primaria universal, con un porcentaje alto los que la han alcanzado y otros están en vías de lograrlo. Este panorama favorable, sin embargo, es dispar entre los países y en algunos casos lento, por lo cual las brechas de desigualdad e inequidades educativas continúan siendo una historia sin fin para la población en la franja de la pobreza y la pobreza extrema. En la agenda educativa global la respuesta a esta problemática es la equidad social educativa, entendida como la implementación de acciones de políticas públicas de afirmación positiva para los colectivos vulnerables o más desfavorecidos, que en su mayoría son los excluidos del disfrute de los bienes púbicos, entre éstos la educación. En este trabajo se asume equidad social educativa en contraposición a la extendida idea de considerarla sinónimo de igualdad de oportunidades. Para ello, se adoptan, entre otros, los enfoques analíticos de los estudios de EGREES (2015) de la Comunidad Europea; Tiana Ferrer (2007), Dubet (20011) y Marchesi (2000), que aún cuando utiliza el concepto de igualdad de oportunidades, clarifica sus limitantes y alcances. En ese contexto, desde una visión comparada, el análisis comprende los enfoques de política social educativa en las leyes educativas de los 19 países de América Latina como respuestas legislativas de los gobiernos de la región para revertir la exclusión económica y social en la educación, específicamente los planteamientos para su consecución (programas, acciones, mecanismos, incentivos y grupos destinatarios).

\section{Abstract}

Several international organizations (ECLAC, 2010, World Bank, 2010 and Inter-American Development Bank, 2016) place Latin America as the most unequal region in the world, whose effects are translated in an important way in education. It is true that, in general terms, Latin American countries register significant advances in universal primary education, with a high percentage of those that have reached it and others are in the process of achieving it. This favorable outlook, however, is uneven among countries and, in some cases, slow, so that gaps in educational inequality and inequalities continue to be an endless history for the population in the fringe of poverty and extreme poverty. In the global educational agenda, the answer to this problem is educational social equity, understood as the implementation of positively affirmed public policy actions for vulnerable or more disadvantaged groups, which are mostly excluded from the enjoyment of public goods, among them education. This work assumes educational social equity as opposed to the widespread idea of considering it equal of equal opportunities. For this, the analytical approaches of the studies of EGREES (2015) of the European Community are adopted, among others; Tiana Ferrer (2007), Dubet (20011) and Marchesi (2000), that even when using the concept of equal opportunities, clarifies its limitations and scope. In this context, from a comparative perspective, the analysis includes the approaches of educational social policy in the educational laws of the 19 countries of Latin America as legislative responses of the governments of the region to reverse the economic and social exclusion in education, specifically the approaches for its achievement (programs, actions, mechanisms, incentives and target groups).

Palabras clave: exclusión educativa, equidad, igualdad de oportunidades, legislaciones educativas y Latinoamérica.

Keywords: educational exclusion, equity, equal opportunities, educational legislations and Latin America. 


\section{INTRODUCCIÓN}

En general, aunque de manera desigual, los países de América Latina registran progresos significativos, por ejemplo, en la enseñanza primaria universal cuyos datos disponibles estiman que casi tres de cada cinco países la habrían logrado o estaban cerca de la alcanzarla (UNESCO, 2015) y en su mayoría la paridad de género en ese nivel educativo. Pero, la complejidad del mundo moderno globalizado y las persistentes desigualdades socioeconómicas en la región se conjugan para imposibilitar o dificultar que un sector importante de la población desfavorecida ingrese, permanezca y finalice la educación, para que entre otras cuestiones, se beneficie por igual de su potencial, principalmente en la superación de la pobreza y evitar su transmisión inter-generacional, dado el círculo virtuoso existente entre educación, movilidad socio-ocupacional y mejores ingresos (CEPAL, 2010).

En la literatura especializada, los estudios y las evidencias empíricas disponibles confirman la estrecha relación de la segmentación socioeconómica prevaleciente, el lugar de residencia (zonas rurales e indígenas), la etnia, el género y la discapacidad, entre otras características, con la exclusión y las desigualdades en el acceso, permanencia y egreso de la educación. En este contexto, la pobreza está en el centro del círculo de las inequidades educativas. Las cifras adversas no dejan lugar a dudas y basten unos datos:

La incidencia de la pobreza y de la pobreza extrema es más elevada en la población que reside en áreas rurales; donde en 2016, la pobreza afectaba al 46,7\% de los niños y adolescentes entre 0 y 14 años y la extrema pobreza al $17 \%$ y en los jóvenes de 15 a 29 años era de $31,1 \%$ y $9,5 \%$, respectivamente. Además, esta problemática parece imparable, toda vez que los niveles de pobreza y pobreza extrema aumentaron en América Latina como promedio regional en 2015 y 2016, después de más de una década de reducción en la mayoría de los países; mientras que en 2017 se mantendrían estables. En 2014, 28,5\% de la población de la región se encontraba en situación de pobreza (168 millones de personas), porcentaje que aumentó a $29,8 \%$ en 2015 (178 millones) y a 30,7\% en 2016 (186 millones de personas). La pobreza extrema, en tanto, pasó del 8,2\% en 2014 (48 millones de personas) al 10\% en 2016 (61 millones de personas) (CEPAL, 2017, s/p).

No obstante, el avance favorable en la enseñanza primaria universal y los Programas de Transferencias Condicionadas (PTC) vinculados directamente a la educación y destinados a la población pobre y en extrema pobreza, han sido insuficientes para los sectores vulnerables ante las persistentes desigualdades de origen, particularmente el socioeconómico. Las cifras disponibles también lo confirman: cerca de 3 millones de niños en edad escolar están fuera de la escuela en la educación primaria; una elevada tasa de varones no participa en la educación secundaria, de la cual $60 \%$ de la población mayor de 18 años no tiene completo este nivel y poca más de 28 millones de jóvenes y adultos de 15 años de edad y más (55\% mujeres) son analfabetos (UNESCO, 2017 y 2015). Otro dato revelador: en promedio, en América Latina $17.3 \%$ de la población joven urbana entre 15 y 24 años de edad no estudian ni trabajan, siendo mayor en los jóvenes de las zonas rurales con $21.7 \%$ (CEPAL, 2016) ${ }^{1}$.

Actualmente, la problemática de la exclusión/inclusión constituye un tema central de la agenda educativa mundial, donde la equidad está considerada la vía cardinal para disminuir las brechas de desigualdad educativa y social existentes entre los grupos sociales (UNESCO, 2010). El Banco Mundial (2010) así sintetiza el problema: «El terreno del juego está disparejo desde el principio, por tanto el problema no solo es de igualdad sino de equidad» (p.1).

Bajo esa premisa, el objetivo general de este trabajo es analizar los planteamientos de política social educativa en las leyes educativas de los 19 países de América Latina dirigidos a los colectivos desfavorecidos. El análisis parte de asumir dos premisas: una, las perspectivas conceptuales de la agenda global de UNESCO (2005) que desde este año estableció la necesidad de integrar políticas educativas (equidad educativa) con otras económicas y sociales (equidad social), dado que los sistemas educativos no son una isla y por sí mismos no pueden romper las brechas socioeconómicas y culturales entre los escolares, pero incluso, las pueden estar reproduciendo o aumentando. La segunda, es diferenciar equidad social educativa de igualdad de oportunidades, que frecuentemente se les ha considerado sinónimos, pero una y otra tienen diferentes implicaciones conforme a los estudios 
de EGREES (2005), Tiana Ferrer (2007) Dubet (2011) y Sen (1992), entre otros. Aquí, se reconoce, que ambas son cara de una misma moneda y si bien la igualdad de oportunidades es un primer nivel importante o piso mínimo hacia la equidad, por sí misma es insuficiente para una equidad socioeducativa dada la vulnerabilidad de origen de las personas en lo económico, social, lugar de residencia, étnico, género, etcétera.

El estudio de EGREES resulta esclarecedor al distinguir entre equidad e igualdad de oportunidades, en función del cuestionamiento de Sen (1992) " ¿igualdad de qué?», cuando juzga que el principio jurídico de igualdad resulta una «igualdad hipotética o formal». Es decir, una visión estrictamente igualitaria dando el mismo trato a todos ignorando las iniciales características individuales de las personas obliga a cuestionar sus fundamentos, precisamente por razones de igualdad (EGREES, 2005 y Dubet, 2011). En el ámbito jurídico, la justicia y la igualdad tienen una connotación vinculante, cuyo postulado establece que todas las personas son iguales ante la ley y nadie puede ser privado de lo que por derecho o justicia le corresponde (Bracho y Hernández, 2006:3). Para Blázquez, Devesa y Cano (2002) este principio jurídico de igualdad formal, se ha tratado de compensar con el «voluntarioso concepto de igualdad de oportunidades, que no suele traspasar los límites de las buenas intenciones». Al respecto, EGREES (2005) ejemplifica que aun aceptando que un sistema educativo es justo, al tratar a todos los alumnos de igual a igual y los activos esenciales se distribuyan con reglas de la justicia y en pie de igualdad -por ejemplo, profesores de idéntica calidad- otros activos se distribuyen en proporción a la compensación y la recompensa (meritocracia individual de talento o habilidades). Por tanto, concluye EGREES (2005) que un «enfoque estrictamente igualitario es imposible» por lo que se deben tener en cuenta la multiplicidad de principios de la justicia y no uno sólo» (p.15).

Los cuestionamientos a la igualdad de oportunidades son más entendibles al analizar sus imperativos básicos: educación pública, gratuita y obligatoria -en la mayoría de los países comprende educación primaria y secundaria- que con todo y sus potencialidades no resultan equitativos para todos los colectivos desfavorecidos. Un ejemplo útil que convalida este planteamiento es la gratuidad educativa, aplicable también a los otros dos imperativos. Es decir, tal como lo plantea la UNESCO (2005) el hecho de que la educación sea gratuita no implica que todos los ciudadanos de un país puedan acceder a ella. Simplemente, por un lado, el costo de insumos como útiles y ropa escolares, transporte, materiales educativos; y por el otro, la disponibilidad de planteles educativos cercanos o accesibles al lugar de residencia; como también los ambientes socioculturales de los escolares, la pertenencia a una minoría étnica, familias inmigrantes o sin vivienda adecuada, desconocimiento del lenguaje mayoritario, tipo de escuela y falta de apoyo social, entre otros. Para la CEPAL (2018) un determinante común de los problemas causados por la desigualdad es la ausencia o la baja calidad de bienes públicos, entre ellos la educación, como también la carencia de sistemas de reglas que garanticen la igualdad de oportunidades.

Incluso un autor como Marchesi (2000), que utiliza el término de igualdad de oportunidades, clarifica sus limitaciones y alcances en los siguientes términos: [...] a) un «primer nivel» es la igualdad de acceso al tener todos los alumnos formal y legalmente las mismas posibilidades educativas y éstas son accesibles a todos sin criterios encubiertos de acceso y de selección b) un "nivel superior» es cuando garantizada la igualdad en el acceso, se proporciona un programa educativo similar a todos los alumnos evitando que quienes proceden de clases sociales populares estén mayoritariamente representados en los programas menos valorados social y académicamente; y c) el «significado más fuerte» de igualdad educativa se encuentra al analizar si los resultados de aprendizaje de los alumnos son iguales o similares entre los escolares procedentes de distintas clases sociales, culturas o sexos (p.136). Esto no implica resultados homogéneos, pero sí que los estudiantes adquieran y egresen con las competencias básicas (Tiana, 2007), para insertarse en el mercado laboral y/o la continuación de estudios, según sea el caso.

Ahora bien, los temas de la inclusión/exclusión y/o la equidad/inequidad en la educación han sido discutidos en diversos trabajos, en el ámbito internacional y regional latinoamericano, por diversos organismos y teóricos en el terreno académico, por lo cual cabe preguntarse por qué un trabajo más. La respuesta es simple, por dos razones: la publicitación de la agenda 20/30: una nueva visión de la educación ( $4^{\circ}$. Objetivo de Desarrollo Sostenible) y el enfoque del Informe de Seguimiento de la Educación en el Mundo 2017/2018 (UNESCO, 2017) cuyo título es revelador: «Rendir cuentas en el ámbito de la educación. Cumplir con nuestro compromiso». 


\section{EQUIDAD SOCIALEDUCATIVA EN LAS LEYES EDUCATIVAS DE AMÉRICA LATINA}

Tabla 1. CATEGORÍAS DE EQUIDAD SOCIAL EDUCATIVA EN LAS LEYES EDUCATIVAS DE AMÉRICA LATINA

\begin{tabular}{|c|c|c|c|c|c|c|c|c|c|}
\hline \multicolumn{10}{|c|}{ EQUIDAD SOCIAL EDUCATIVA } \\
\hline \multirow{3}{*}{ 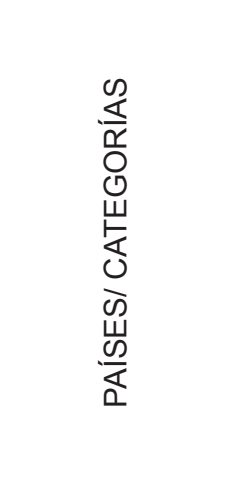 } & \multicolumn{2}{|c|}{$\begin{array}{c}\text { ENFOQUE DE } \\
\text { POLIITICA EDUCTIVA }\end{array}$} & \multicolumn{4}{|c|}{ IGUALDAD DE OPORTUNIDADES } & \multicolumn{3}{|c|}{$\begin{array}{l}\text { ACCIONES DE EQUIDAD } \\
\text { SOCIAL EDUCATIVA }\end{array}$} \\
\hline & \multirow[b]{2}{*}{ 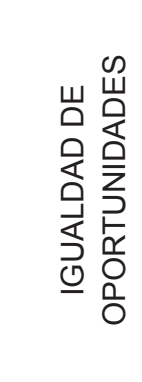 } & \multirow[b]{2}{*}{ 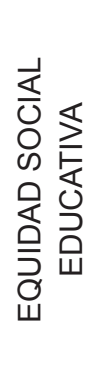 } & \multirow[b]{2}{*}{ 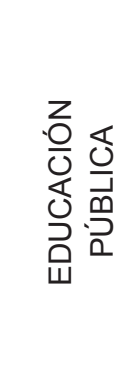 } & \multirow[b]{2}{*}{ 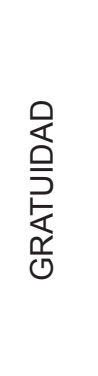 } & \multicolumn{2}{|c|}{ OBLIGATORIEDAD } & \multirow{2}{*}{ 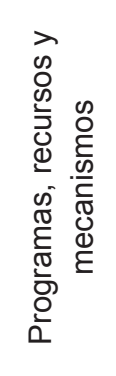 } & \multirow{2}{*}{ 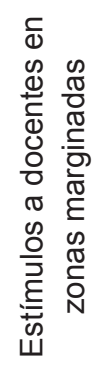 } & \multirow{2}{*}{ 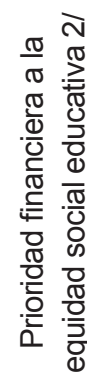 } \\
\hline & & & & & 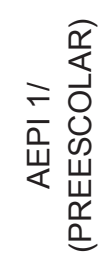 & 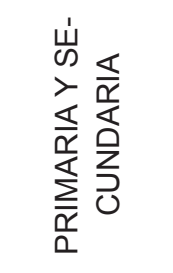 & & & \\
\hline ARGENTINA & $\checkmark$ & $\checkmark$ & $\checkmark$ & $\checkmark$ & $\checkmark$ & $\checkmark$ & $\checkmark$ & $\checkmark$ & $\checkmark$ \\
\hline BOLIVIA & $\checkmark$ & $\checkmark$ & $\checkmark$ & $\checkmark$ & $\checkmark$ & $\checkmark$ & $\checkmark$ & - & - \\
\hline BRASIL & $\checkmark$ & - & $\checkmark$ & $\checkmark$ & $\bullet$ & $\checkmark$ & $\checkmark$ & - & - \\
\hline CHILE & $\checkmark$ & $\checkmark$ & \multicolumn{2}{|c|}{$\varnothing$} & $\bullet$ & $\checkmark$ & - & - & - \\
\hline COLOMBIA & - & - & $\checkmark$ & $\checkmark$ & $\checkmark$ & $\checkmark$ & $\downarrow$ & - & - \\
\hline COSTA RICA & - & - & $\checkmark$ & $\checkmark$ & $\checkmark$ & $\checkmark$ & - & - & - \\
\hline CUBA & $\checkmark$ & - & \multicolumn{2}{|c|}{$\begin{array}{l}\text { Toda la educación } \\
\text { es pública y gratuita }\end{array}$} & $\bullet$ & $\checkmark$ & - & - & - \\
\hline ECUADOR & $\checkmark$ & $\checkmark$ & $\checkmark$ & $\checkmark$ & $\checkmark$ & $\checkmark$ & $\checkmark$ & $\checkmark$ & $\checkmark$ \\
\hline EL SALVADOR & - & & $\checkmark$ & $\checkmark$ & $\checkmark$ & $\checkmark$ & - & - & - \\
\hline GUATEMALA & - & - & $\checkmark$ & $\checkmark$ & $\checkmark$ & $\checkmark$ & $\checkmark$ & $\checkmark$ & - \\
\hline HONDURAS & $\checkmark$ & $\checkmark$ & $\checkmark$ & $\checkmark$ & $\checkmark$ & $\checkmark$ & $\checkmark$ & - & - \\
\hline MÉXICO & $\checkmark$ & $\checkmark$ & $\checkmark$ & $\checkmark$ & $\checkmark$ & $\checkmark$ & $\checkmark$ & $\checkmark$ & - \\
\hline NICARAGUA & $\checkmark$ & $\checkmark$ & $\checkmark$ & $\checkmark$ & $\checkmark$ & PRIMARIA & $\checkmark$ & $\checkmark$ & $\checkmark$ \\
\hline PANAMÁ & - & & $\checkmark$ & $\checkmark$ & $\checkmark$ & $\checkmark$ & $\checkmark$ & - & - \\
\hline PARAGUAY & $\checkmark$ & $\checkmark$ & $\checkmark$ & $\checkmark$ & $\checkmark$ & $\checkmark$ & $\checkmark$ & - & - \\
\hline PERÚ & $\checkmark$ & $\checkmark$ & $\checkmark$ & $\checkmark$ & $\checkmark$ & $\checkmark$ & $\checkmark$ & $\checkmark$ & $\checkmark$ \\
\hline $\begin{array}{c}\text { REP. } \\
\text { DOMINICANA }\end{array}$ & $\checkmark$ & $\checkmark$ & $\checkmark$ & $\checkmark$ & $\checkmark$ & $\checkmark$ & $\checkmark$ & - & - \\
\hline URUGUAY & $\checkmark$ & $\checkmark$ & $\checkmark$ & $\checkmark$ & $\checkmark$ & $\checkmark$ & $\checkmark$ & - & - \\
\hline \multirow[t]{3}{*}{ VENEZUELA } & $\checkmark$ & & $\checkmark$ & $\checkmark$ & $\checkmark$ & $\checkmark$ & - & - & - \\
\hline & & & $18 \checkmark$ & $18 \checkmark$ & $16 \checkmark$ & $18 \checkmark$ & $13 \checkmark$ & $5 \checkmark$ & $5 \checkmark$ \\
\hline & & & $1 \varnothing$ & $1 \varnothing$ & 3 & 1 PRIMARIA & $1 \downarrow$ & $14-$ & $14-$ \\
\hline TOTAL LEYES & \multicolumn{2}{|c|}{16} & 19 & 19 & 19 & 19 & 14 & $5 \checkmark$ & $5 \checkmark$ \\
\hline
\end{tabular}

Fuente: Elaboración propia con base en: Análisis de las 19 Leyes Generales de Educación de América Latina.

Notas:

- Categorías no reguladas

- Débil alusión de equidad

$\varnothing$ En su ley educativa Chile declara la educación como pública, pero en la realidad es privada

1/ AEPI (Atención y Educación a la Primera Infancia). En la Tabla, la obligatoriedad corresponde al preescolar.

"No es obligatorio el preescolar

$\downarrow$ Las acciones de políticas públicas de equidad social educativa son selectivas.

2/ Se refiere únicamente a la prioridad presupuestal para las acciones educativas y docentes en la educación de los grupos desfavorecidos y zonas marginada. 


\subsection{Enfoque de política educativa}

Como puede observarse en la Tabla 1 precedente, resulta positivo que 16 leyes tengan un enfoque de política educativa (de las cuales tienen un bajo perfil las de El Salvador, Panamá y Venezuela), sea como igualdad de oportunidades o equidad social educativa, y en algunos casos ambas. La excepción son las de Costa Rica (la más deficitaria), Colombia y Guatemala que no especifican esa categoría, aunque las dos últimas establecen acciones de equidad social educativa (programas, recursos y mecanismos).

\subsubsection{Igualdad de oportunidades}

Los 19 países latinoamericanos convergen en la igualdad de oportunidades con los imperativos del carácter público, gratuito y obligatorio de la educación preescolar, primaria y secundaria, aunque en Brasil, Cuba y Chile el preescolar no es obligatorio y en Nicaragua la secundaria. En los polos dispares entre los países en la educación pública y gratuita está Chile -donde los servicios educativos son privados (UNESCO, 2010/211) - cuya ley si bien estipula no afectar durante el ciclo escolar a los alumnos por el no pago de aranceles y matrícula, autoriza el posterior cobro a los «sostenedores», que son los particulares a quienes subvenciona la prestación de la educación; en contraste, en Cuba toda la educación es pública y gratuita en todos sus niveles educativos. No obstante, sin duda, un avance legislativo de la mayor relevancia a favor de la equidad es que 16 leyes norman el nivel inicial o Atención y Educación a la Primera Infancia (AEPI) también con ciertas limitantes y dimensiones. Es pertinente mencionar que las leyes educativas de Cuba, Colombia y Costa Rica no regulan el nivel inicial o AEPI, pero sí forma parte de su política educativa o de sus sistemas educacionales (UNESCO, 2010/211).

\subsubsection{Programas, recursos y mecanismos}

Con mayor o menor detalle, algunas leyes educativas destacan en estipular una serie planteamientos de equidad social educativa, a través de acciones de políticas públicas (programas, recursos, incentivos, etcétera) a efecto de garantizar a los colectivos desfavorecidos el acceso, la permanencia en los servicios educativos y la conclusión de los estudios (el egreso).

La de México es de las más profusas, cuya política social del Estado comprende una serie de medidas para contrarrestar las condiciones sociales que inciden en la efectiva igualdad de oportunidades de acceso y permanencia en los servicios educativos. Responsabiliza a las autoridades educativas desarrollar una política social consistente en: promover centros de desarrollo infantil, integración social, internados, albergues escolares e infantiles y otros planteles de apoyo al aprendizaje y aprovechamiento de los alumnos; servicios educativos para atender a quienes abandonaron estudios; en rezago educativo y concluyan la educación básica, especialmente a las mujeres; apoyos pedagógicos a grupos con requerimientos educativos específicos; becas y apoyos económicos preferentemente a los estudiantes en condiciones económicas y sociales que les impidan ejercer su derecho a la educación; materiales educativos en las lenguas indígenas en las escuelas con población mayoritariamente indígena.

Igualmente destaca la de Argentina al estipular acciones en las zonas rurales con programas de becas, comedores escolares y otros servicios asistenciales a la comunidad. La educación de adultos comprende servicios educativos presenciales y a distancia, becas para jóvenes y adultos, provisión gratuita de materiales de aprendizaje y asegurar su permanencia y egreso. En el capítulo «Políticas de promoción de la igualdad educativa» establece enfrentar situaciones de injusticia, marginación, estigmatización y otras formas de discriminación, por factores socioeconómicos, culturales, geográficos, étnicos, género o de cualquier otra índole, que afecten el ejercicio pleno del derecho a la educación y aseguren las condiciones necesarias para la inclusión, el reconocimiento, la integración y el logro educativo de todos/as los/as niños/ as, jóvenes y adultos en todos los niveles y modalidades, principalmente los obligatorios.

Con similar política social la ley de Bolivia determina implementar programas sociales específicos de los/las estudiantes desfavorecidos económicamente, mediante recursos financieros, programas de alimentación, vestimenta, transporte y material escolar. En áreas dispersas becas a las/los estudiantes de excelente aprovechamiento en todos los niveles educativos. Establece la educación escolarizada integral para la población en desventaja social dirigida a la atención de niñas/os y adolescentes, jóvenes trabajadores desprotegidos con programas especiales de hogares abiertos servicios integrales de salud, alimentación, educación, reinserción escolar y socio-laboral, y dar prioridad al rezago escolar. 
La de Ecuador también profusa, con medidas compensatorias como: garantizar el acceso universal a la educación con políticas y programas especiales y recursos para facilitar la escolarización regular de niñas/os y adolescentes que por inequidad social presenten dificultades de inserción educativa, desfase escolar o por cualquier motivo demanden intervenciones compensatorias por su incorporación tardía a la educación. Adoptar medidas que favorezcan a segmentos sociales en situación de abandono o riesgo, para compensar las desigualdades por factores económicos, geográficos, sociales o de cualquier otra índole, con atención especial al a personas excluidas del sistema educativo con becas y apoyos económicos para su acceso en igualdad de condiciones a la educación, con prioridad a las mujeres.

Un grupo de leyes resalta por establecer también programas de provisión de diversos insumos educativos, alimenticios o nutricionales. La de Brasil, dispone la atención gratuita y obligatoria en guarderías y centros preescolares a los niños de cero a seis años de edad; atención al educando en la enseñanza fundamental con programas suplementarios de material didáctico-escolar, transporte, alimentación y asistencia a la salud; oferta de enseñanza nocturna regular y educación especial a los portadores de deficiencias, preferentemente en el sistema ordinario de enseñanza. En la misma línea, la de Nicaragua establece llevar a cabo programas asistenciales, ayudas alimenticias, campañas de salubridad y demás medidas para contrarrestar las condiciones sociales que inciden en la efectiva igualdad de oportunidades de acceso y permanencia en la educación y programas compensatorios en entidades o estratos con mayores rezagos educativos.

La de República Dominica a través del Instituto Nacional de Bienestar Estudiantil promueve la participación de los estudiantes a organizar servicios de transporte, nutrición escolar y salud, apoyo de materiales y útiles escolares, becas y trabajo remunerado en vacaciones, suministro regular y gratuito del desayuno escolar a todos los niños/as del sistema educativo que los necesiten. La de Paraguay, en el capítulo «De la compensación de las desigualdades en la educación», plantea "extender progresivamente la gratuidad» de los programas de complemento nutricional y el suministro de útiles escolares para los alumnos de escasos recursos y facilitar su ingreso en los establecimientos públicos gratuitos. Pero, ante la carencia o insuficiencia de planteles educativos para atender a colectivos en situación económica desfavorable, establece que el Estado financie plazas de estudios en los centros privados, con becas, parciales o totales.

La de Guatemala determina para los estudiantes de los niveles educativos obligatorios otorgar de manera gratuita útiles escolares, «bolsas» de estudios, becas, créditos y otros beneficios, desarrollar programas para mejorar su salud, nutrición y recreación. Las becas son para estudiantes con «vocación», por rendimiento escolar, aptitudes y/o no contar con los medios económicos para sostener sus estudios. La de Perú entre otras medidas para la equidad plantea: políticas compensatorias de acción positiva y proyectos educativos, para compensar las desigualdades de la población necesitada. En la educación inicial y primaria señala programas obligatorios de alimentación, salud y materiales educativos; mecanismos para la matrícula oportuna, la permanencia y la reincorporación de los estudiantes a la educación y medidas de retención ante el riesgo de exclusión del servicio; becas y ayudas para garantizar acceder o continuar los estudios alumnos destacados en su rendimiento académico y desfavorecidos económicamente para cubrir los costos de su educación.

La de Honduras determina para los niños de padres de escasos recursos económicos programas de entrega de uniformes, zapatos y útiles escolares gratuitos de los niveles pre-básico, básico y medio, cuyas autoridades educativas y desarrollo social firmarán convenios con instituciones públicas o privadas. La de Panamá establece otorgar becas y asistencias económicas a los alumnos de bajos recursos económicos, de buen rendimiento académico y discapacitados en todos los niveles educativos. En cambio, la de Colombia con una orientación economicista y selectiva prescribe otorgar subsidios y "créditos» a las familias de menores ingresos económicos para el pago de diversos gastos escolares en escuelas estatales o privadas. La de El Salvador determina fomentar programas de becas, subvenciones y "créditos financieros», para quienes teniendo capacidad intelectual y aptitud vocacional, aspiren a estudios superiores a la educación básica.

\subsubsection{Estímulos a docentes en las zonas marginadas}

Aspecto de importancia vital de la equidad vinculado a la calidad educativa, es la renuencia de docentes de trabajar en las zonas marginadas o desfavorecidas, situación que únicamente 5 leyes contrarrestan. La de Argentina menciona diseñar estrategias para que los/as docentes con mayor experiencia y calificación se desempeñen en las escuelas más desfavorables. La de México enuncia programas de apoyo a maestros que trabajen en localidades aisladas o zonas urbanas marginadas, para fomentar el arraigo en sus comunidades y cumplir con el calendario escolar. La de Ecuador establece bonificación para los docentes en zonas de difícil acceso. 
La de Perú consigna desarrollar programas de bienestar y apoyo técnico para la permanencia de los maestros en las zonas rurales, de menor desarrollo y socialmente vulnerables, y donde sea pertinente dar incentivos salariales, de vivienda y otros. La de Nicaragua establece incrementos anuales a los docentes para el mejoramiento de su nivel de vida, aunque considerando las limitaciones presupuestales.

\subsubsection{Prioridad financiera a la equidad social educativa}

Únicamente 5 leyes disponen priorizar la asignación de los recursos económicos para asegurar la igualdad de oportunidades y la equidad educativa. La de Perú determina la asignación de recursos por alumno en las zonas de mayor exclusión, para infraestructura, equipamiento, material educativo y recursos tecnológicos. La de Ecuador establece que la asignación y distribución de los recursos destinados a la educación «combina y articula los principios constitucionales de equidad social, poblacional y territorial». La de Paraguay menciona dar prioridad a la asignación de recursos a la educación de los sectores marginales de la población, sector rural, las áreas urbanas marginales y las zonas fronterizas. La de Nicaragua, aunque de forma limitativa, dispone que el gobierno incremente anualmente el presupuesto de la educación no superior considerando calidad, equidad y crecimiento de la cobertura escolar, construcción, mantenimiento de infraestructura. La de Argentina dispone la asignación con equidad de los recursos económicos en la educación.

\section{DISCUSIÓN Y CONCLUSIONES}

Es bien cierto que a las leyes educativas no se les puede pedir más allá de lo que ofrecen, pero si exigir a los gobiernos que cumplan a lo que se comprometen en ellas, como tampoco se da en automático lo que está prescrito en una normativa jurídica, ni se puede soslayar las discrepancias y tensiones entre las leyes y la práctica. Tampoco, las políticas públicas son panacea per se para la solución de todos los problemas públicos, pero sí contribuyen a hacerlos más manejables despojándolos de sus aspectos más nocivos.

El análisis de las leyes educativas de América Latina refleja la heterogeneidad de los países en materia de política social educativa; aunque es destacable que las 19 normativas por lo menos consideran la igualdad de oportunidades, cuyos cuestionamientos tampoco la invalidan como necesaria para la equidad social educativa. El déficit más importante son los incentivos a docentes y priorizar el presupuesto para la educación de los colectivos vulnerables y las zonas marginadas (apenas 5 leyes lo contemplan). Al mismo tiempo, la región está a la vanguardia legislativa al regular la atención a la primera infancia y el preescolar, dos asuntos ausentes en los tratados internacionales de derechos humanos como componentes del derecho a la y en la educación. Sin embargo, en la región alrededor de 2015, la media de la tasa bruta de matriculación en atención y desarrollo de la primera infancia y acceso a preescolar fue de $42 \%$.

Para avanzar hacia la equidad social educativa, es de capital importancia corregir desde su génesis las desigualdades, esto es, las condiciones iniciales de vida e incluso aún antes de éstas; pues juegan un papel fundamental en las situaciones de exclusión y en la desvinculación socioeducativa, con consecuencia en la ausencia de perspectivas inmediatas y futuras para los sectores desfavorecidos. Además, esto genera y perpetúa una mayor vulnerabilidad con consecuencias diversas (violencia, drogadicción, delincuencia, entre otros problemas), problemáticas éstas y otras generadas por la «ineficiencias de la desigualdad» que son un obstáculo para el desarrollo sustentable de los países en lo económico, social, político, educativo, ambiental, productivo, entre otros ámbitos (CEPAL, 2018).

Ciertamente, para encarar los desequilibrios inclusión versus exclusión es necesario cuestionar los esquemas actuales en la inequitativa distribución en cuanto a recursos (financieros, humanos e infraestructura, entre otros), los procesos (acceso, deserción, reprobación y eficiencia terminal) y los resultados (logro académico y tasas de graduación, por ejemplo). En ambo casos, en articulación con las políticas públicas para que en conjunto proporcione un sustento adecuado para la toma de decisiones y acciones de los diversos actores involucrados y los objetivos educativos sean alcanzados por el mayor número posible de las personas en la región.

No es posible en este trabajo hacer un análisis empírico profundo de esas inequidades educativas sociales, pero las cifras aquí presentadas son la punta del iceberg de la problemática, para evidenciar 
que la exclusión educativa e incluso, en algunos países, la igualdad de oportunidades, continúan siendo una historia sin fin en América Latina. Por tanto, los países de la región tienen el reto de pasar del lenguaje legislativo a cumplir, hacer efectivas o implementar, según sea el caso, acciones específicas de política social educativa de afirmación positiva, para neutralizar o cuando menos aminorar las desigualdades educativas en el acceso, la permanencia y el egreso de la educación, así como la calidad y los resultados de aprendizaje. En consecuencia, no se puede tratar igual a los desiguales, pues se estaría ante un derecho a la educación para unos y negado para otros. Esto implica, que los Estados realicen su política educativa con prestaciones reales y accesibles a todas las personas de los bienes públicos, reconociendo la diversidad en la igualdad, para generar procesos de cambio y mejora educativa en beneficio de los colectivos vulnerables.

\section{REFERENCIAS BIBLIOGRÁFICAS}

Banco Interamericano de Desarrollo (2016): Pulso Social de América Latina y el Caribe 2016: realidades y perspectivas / Duryea, S. y Robles, M., Banco Interamericano de Desarrollo. Recuperado de: https://publications.iadb.org/handle/11319/7863? locale-attribute=es\&.

Banco Mundial (2010): Do Our Children Have A Chance? The 2010 Human Opportunity Report for Latin America and the Caribbean. Recuperado de: www.worldbank.org.

Blázquez F., Devesa A. y Cano, M. (2002): Diccionario de términos éticos, Navarra: Editorial Verbo Divino.

Bracho, T. y Hernández, J. (2006): Equidad Educativa. Avances en la definición de su concepto. En: Memorias del X Congreso Nacional de Investigación Educativa, Interrelaciones educación-sociedad, México: Universidad Veracruzana

Comisión Económica para América Latina y el Caribe (2018): La ineficiencia de la desigualdad, 2018. Recuperado de: https:// periododesesiones.cepal.org/37/es/documentos/la-ineficiencia-la-desigualdad.

(2017): Presentación del Panorama Social en América Latina [Conferencia de prensa]. Recuperado de: https:// www.cepal.org/es/comunicados/.

(2016): Jóvenes de 15 a 24 años de edad que no estudian ni trabajan (o no integran la PEA), según grupos de edad y por área geográfica [Información revisada al 25 de mayo de 2016 por la CEPAL]. Recuperado de: http:// estadisticas.cepal.org/cepalstat/web_cepalstat/estadísticas Indicadores.asp?idioma=e.

(2010): La hora de la igualdad. Brechas por cerrar, caminos por abrir, Recuperado de: http://www.eclac.org/.

Dubet, F. (2011): Repensar la Justicia Social, Serie Educación y Sociedad (1ª edición español) (Traducción de Alfredo Grieco y Bavio), Buenos Aires: Siglo XXI Editores Argentina, S. A.

European Group of Research on Equity of the Educational Systems (EGREES, 2005): Equity in European Educational Systems. A Set of Indicators. En: European Educational Reserch Journal, volumen 4, núm. 2.

Leyes educativas de América Latina. Recuperadas de http://www.oei.es/historico/quipu/legislacion_educativa.htm.

Marchesi, A. (2000): Un sistema de indicadores de desigualdad educativa. En: Revista Iberoamericana de Educación (Número 23), Madrid: Organización de Estados Iberoamericanos.

Sen, A. (1992): Nuevo examen de la desigualdad, Madrid: Alianza.

Tiana, A. (2007): Calidad, equidad e integración educativa. En: Libertad, calidad y equidad en los sistemas educativos (buenas prácticas internacionales), Madrid: Fundación Europea Sociedad y Educación y Comunidad de Madrid.

UNESCO (2017): Informe de Seguimiento de la Educación en el Mundo 2017/08. Rendir cuentas en el ámbito de la educación. Cumplir nuestros compromisos (Primera edición). Recuperado de: (http://www.unesco.org/).

(2015): Panorama Regional: América Latina y el Caribe. Recuperado de: http://unesdoc.unesco.org/images/0023/002328/232836s.pdf.

(2011): Panorámica Regional: América Latina y el Caribe. Recuperado de: http://unesdoc.unesco.org/images/0019/001914/191433s.pdf .

2010/2011: Datos Mundiales de Educación, Buro Internacional de Educación, $7^{a}$ Edición. Recuperado de: http// www.ibe.unesco.org.

(2010): Compendio Mundial de la Educación. Recuperado de http://unesdoc.unesco.org/images/0019/001912/ 191218s.pdf.

(2005): Informe de Seguimiento de la EPT en el Mundo 2005. El Imperativo de la Calidad. Recuperado de: www. efareport.unesco.org. 